\title{
Política de salud y crisis sanitaria frente a la covid-19 del cono norte - Lima
}

\author{
Luis Roberto Malarin Rojas \\ lmalarinro4@ucvvirtual.edu.pe \\ https://orcid.org/0000-0002-4976-3426 \\ Universidad Cesar Vallejo \\ Lima - Perú
}

\section{RESUMEN}

El presente estudio es cuantitativo, método aplicado fue hipotético deductivo tipo básica de corte transversal y correlacional sobre política de salud y crisis sanitaria frente a la Covid-19 del cono norte, la pandemia ha desnudado en el mundo las falencias y crisis del sector salud, el Perú no es ajeno frente a esta situación; proponiéndose: ¿Cuál es la relación entre la política de salud con la crisis sanitaria frente a la Covid-19 del cono norte - Lima? Objeto: Determinar la relación entre política de salud y crisis sanitaria frente a la Covid-19 del cono norte - Lima. Se tomo una muestra a 90 funcionarios del sector salud del cono norte, aplicándose dos instrumentos política de salud y crisis sanitaria, que fueron validados por expertos, para medir la confiabilidad se utiliza el coeficiente alfa, en ambas variables. Concluyendo: Se determina que existe una correlación positiva muy alta entre política de salud y crisis sanitaria frente a la Covid-19 del cono norte - Lima. Se deduce que si se realiza buenas políticas de salud entonces la crisis sanitaria su impacto sería menor en la población.

Palabras clave: política de salud; crisis sanitaria y covid-19. 


\title{
Health policy and health crisis facing covid-19 in the northern cone - Lima
}

\begin{abstract}
The present study is quantitative, the applied method was hypothetical deductive, basic type, cross-sectional and correlational on health policy and health crisis facing the Covid19 in the northern cone, the pandemic has uncovered in the world the shortcomings and crisis of the health sector, Perú is no stranger to this situation; proposing: What is the relationship between health policy with the health crisis facing the Covid-19 in the northern cone - Lima? Objective: To determine the relationship between health policy and the health crisis in relation to Covid-19 in the northern cone - Lima. A sample of 90 health sector officials from the northern cone was taken, applying two instruments, health policy and health crisis, which were validated by experts, to measure reliability using the alpha coefficient in both variables. Conclusion: It is determined that there is a very high positive correlation between health policy and health crisis in relation to the Covid-19 of the northern cone - Lima. It is deduced that if good health policies are implemented, then the health crisis would have less impact on the population.
\end{abstract}

Keywords: health policy; health crisis and covid-19.

Artículo recibido: 30 noviembre. 2021 Aceptado para publicación: 29 diciembre 2021 Correspondencia: 1malarinro4@ucvvirtual.edu.pe

Conflictos de Interés: Ninguna que declarar 


\section{INTRODUCCIÓN}

Frente a esta situación vivido mundialmente suscitado en la actualidad por la presencia del virus llamado Covid-19 que ha generado una catástrofe en el ámbito de salud ocasionando crisis hospitalaria en todos los países. La mayor parte de los problemas de salud frente a la crisis por la llegada de la pandemia a nuestro país se pueden imputar al factor socios - económicos en la ciudadanía. Por consiguiente, una política de salud ha tenido siempre como prioridad las enfermedades pares las soluciones diversas en crisis sanitaria que afectan a las personas. Estos problemas sanitarios han ido en incremento, la inequidad y atenciones sanitarias en salud han sido insuficientes cuyo resultado obtenido con intervención de la salud en frente a la covid-19 han sido catastróficas. Ibáñez (2020) menciona que la pandemia ha traído cuantiosas pérdidas en los gobiernos.

El gobierno ha tomado diferentes medidas paliativo en el ámbito de políticas de salud a fin de fortalecer sus instituciones y suplir las necesidades en tiempos de pandemia. El rápido avance del covid_19 ha hecho que todo colapse empezando por la economía y en seguida la crisis hospitalaria destapando una serie de desigualdades entre sus pueblos generando un gran impacto de angustia y desesperación en los hospitales nunca visto y mostrado la cruda y real situación de deficiencia en la gestión de salud pública del Perú. Nuestro país, no es la excepción a éste gran problema puesto que su descuidada Política de Salud a sumido en una crisis sanitaria para enfrentar a esta pandemia que le ha costado al país 200,000 mil muertes, que aún constituyen una aproximación del total de fallecidos Llerena y Sánchez (2020).

La Política Nacional Multisectorial de Salud (PNMS) al 2030, denominado "Perú, País Saludable" son políticas dados por el ministerio de salud, con enfoques territoriales, consensuados por el ciudadano en atención frente a la salud que reciben, durante su vida basados mediante enfoques de "Cursos de Vida", teniendo como aplicación a enfoque y principio de equidad, interculturalidad y derecho en términos de la salud donde el estado debe tener centros de salud que brinden atención frente a las necesidades físicas y económicas accesible favoreciendo un servicio de calidad, con colaboradores médicos de calidad, capacitados, disposición de medicamento, equipos e infraestructuras hospitalarias, adecuados para hacer frente a la crisis sanitarias por la Covid_19.

Las políticas de salud dentro de su rol deben trabajar para reducir al mínimo las brechas de nuestras capacidades especialmente en el área de salud que ha sido sobrepasadas con 
consecuencias fatales en desabastecimiento y sistemas de inversión ineficientes. Las políticas de salud es producto específico del gobierno o cuerpo encomendado por mediante el gobierno, son actividades netamente estatales. Este producto se especifica de modo empírico, mediante listas que tradicionalmente incluyen los sostenimientos de los ingresos, servicio en salud y servicio social, y a veces en educación, viviendas y política de empleos. (Gough, 2003). Teniendo en cuenta, Minsa (2011), referente a política de salud es el marco en la que el individuo de un país se desenvuelve mediante sus comunidades, organizaciones y empresas. Políticas públicas de salud en el Perú, desde la posición Parodi (2005), mediante los nuevos enfoques para tratar la política pública, especialmente la política social de salud, mientras un país crezca económicamente tiene un rol y factores de importancia necesarios, estos proveen el recurso, en pocas palabras, financieras de las políticas sociales. Empleando las palabras de Lahera (2004:8) la política pública es la respuesta en cara a los diferentes problemas que afectan a los ciudadanos. como afirma Knoepfel (2001), el cual refiere a la política pública como proceso concreto que se encaminan a partir de su programación logrando sus objetivos. Tobar (2012) menciona, que son esfuerzos sistemáticos que contribuyen en la resolución de problemas enfocados en la salud.

Desde otro panorama nos enfocas en crisis sanitaria, Como plantea, Testa (1993), "Las crisis son considerados como crisis del estado, objetivadas y parcializadas considerados en ocasiones como crisis sectoriales o transitorias, cuyas resoluciones no admiten solución parcial o coyuntural".

Janssen y van der Voort (2020), manifiesta las crisis sanitarias cambian los sistemas que dominaron el mundo desde 1990, probando que son fenómenos sin precedente y obligan al gobierno en tomar urgentes liderazgos para la adaptación y sobresalir teniendo un liderazgo en adaptarse sobrellevar, adaptar frente a las crisis sanitarias. De acuerdo con Garcia et, al (2020) las autoridades deben tomar decisiones que agreguen valor en tiempos de crisis en salud. A juicio de Olivera et, al (2018), esta crisis también dejo expuesto la carencia de profesionales en salud. Citando a Lamata (2006), La crisis sanitaria ha existido desde muchos años y siempre seguirán existiendo en la humanidad. Toda crisis sanitaria trae consigo problemas que ocasionan daños directos a la salud del ser humano trayendo con ello una serie de riesgo y peligro y atentan con la existencia y salud del ser humano. Por lo tanto, en toda crisis sanitaria aparecerán muchas situaciones nuevas, 
graves derivados de la misma. Información en época de crisis sanitaria. Este reconocimiento hubiese tenido amplias consecuencias con relación al objeto tratado, ya que en el estado de alarma no se pueden suspender los derechos fundamentales y el acceso a la información habría quedado resguardado ante su importancia en un contexto de crisis. Hay que recordar que en estos contextos crece la demanda de información (Westlund y Ghersteti, 2015: 13), pero que se hace más importante aún porque cumple una triple función instrumental (De la Sierra Morón, 2020: 34 y 41): permite el control de la gestión gubernamental, ayuda a "la adopción responsable de decisiones individuales" de los ciudadanos, y contribuye a que otras administraciones públicas puedan actuar conforme a los riesgos definidos. Pero conforme hay demanda de información, las informaciones institucionales y oficiales constituyen las fuentes primordiales en las elaboraciones en noticia, al ser centros de atenciones de referencias principales en consultas, aparte de que ante acontecimientos extraordinarios se incrementan las dificultades de los informadores privados (Ibáñez, 2020).

La problemática abordada es deficiencia en políticas de salud, por lo que la presente investigación está orientada a establecer la relación entre la política de salud y crisis sanitaria frente a la Covid-19 en el cono norte. Ante esta situación se plantea como pregunta general: ¿Cuál es la relación entre la política de salud con la crisis sanitaria frente a la Covid-19 del cono norte - Lima?. Así mismo se plantea como problemas específicos: ¿Cuál es la relación entre la dimensión política de salud equitativa en caso de pandemia con la crisis sanitaria frente a la Covid-19 del cono norte?; ¿Cuál es la relación entre la dimensión política de salud en caso de pandemia con la crisis sanitaria frente a la Covid-19 del cono norte - Lima? y ¿Cuál es la relación entre la dimensión gasto público en salud con la crisis sanitaria frente a la Covid-19 del cono norte - Lima?. Se enfatiza el siguiente objetivo principal: Determinar la relación entre política de salud y crisis sanitaria frente a la Covid-19 del cono norte - Lima, 2020.

Y posteriormente se indican los objetivos específicos: Determinar la relación entre la dimensión política de salud equitativa con la crisis sanitaria frente a la Covid-19 del cono norte - Lima; Determinar la relación entre la dimensión política de salud en caso de pandemia con la crisis sanitaria frente a la Covid-19 del cono norte - Lima;

Determinar la relación entre la dimensión gasto público en salud con la crisis sanitaria frente a la Covid-19 del cono norte - Lima. 
Por lo tanto, esta investigación presenta su justificación teórica sustancial debido al análisis del problema de las políticas de salud y su repercusión frente a crisis sanitaria induciendo a revisión en modelos de política de salud propuestos en muchos países en el mundo y cuál es el mejor indicador para la medición de la crisis de salud en el cono norte. Justificación práctica, esta investigación servirá de aporte a los estudios de la crisis en los sistemas de salud del país tanto en alternativas que se formulan para mejora en política de salud. El estudio contribuirá a dar en conocer cómo se gestionan los hospitales del nivel III-1 de la red de salud, teniendo como referencias, el criterio de política de salud considerando bajo qué medida se puede mejorar indicadores de los desempeños de crisis sanitaria.

Planteando la hipótesis general del estudio: La política de salud se relaciona significativamente con la crisis sanitaria frente a la Covid-19 del cono norte - Lima.

Finalmente mencionamos las hipótesis especificas: La dimensión política de salud equitativa se relaciona significativamente con la crisis sanitaria frente a la Covid-19 del cono norte - Lima; La dimensión política de salud en caso de pandemia se relaciona significativamente con la crisis sanitaria frente a la Covid-19 del cono norte - Lima; La dimensión gasto público en salud se relaciona significativamente con la crisis sanitaria frente a la Covid-19 del cono norte - Lima.

\section{ESTRATEGIAS METODOLÓGICAS O MATERIALES Y MÉTODOS}

El presente estudio es de enfoque cuantitativo, tipo básico, diseño transversal, descriptivo correlacional, donde se identifican las relaciones existentes entre las Políticas de salud y la crisis sanitaria. Diseño de investigación, empleando las palabras de: Hernández et al. (2010, p.81), en el presente estudio, la naturaleza hace que las variables sean consideradas como de diseño descriptivos correlacional. Por lo tanto, se consideran no experimentales al no darse la manipulación de variables. Por tal motivo, la presente investigación corresponde: Diseño no experimental y descriptivo correlacional. Población comprende 116 funcionarios públicos que trabajan en el sector salud de Lima norte. Muestra constituida por 90 funcionarios del sector público que son colaboradores del sector salud del cono norte frente al Covid-19 en Lima-norte. Se utilizo la encuesta tanto para recojo, registro de información para ambas variables, se utilizan técnicas de recolección mediante información proporcionado por el profesional de salud cono norte. Para el tratamiento de los datos se utilizó el software de Microsoft Excel, así como SPSS (25). 


\section{RESULTADOS Y DISCUSIÓN}

\section{Resultados descriptivos}

\section{Tabla 1}

Variable política de salud frente a la Covid-19 del cono norte - Lima.

\begin{tabular}{|c|c|c|c|}
\hline & & Frecuencia & Porcentaje \\
\hline \multirow{4}{*}{ Válido } & Bueno & 13 & 14,4 \\
\hline & Deficiente & 62 & 68,9 \\
\hline & Regular & 15 & 16,7 \\
\hline & Total & 90 & 100,0 \\
\hline
\end{tabular}

\section{Figura 1}

Niveles de la variable política de salud frente a la Covid-19 del cono norte - Lima.

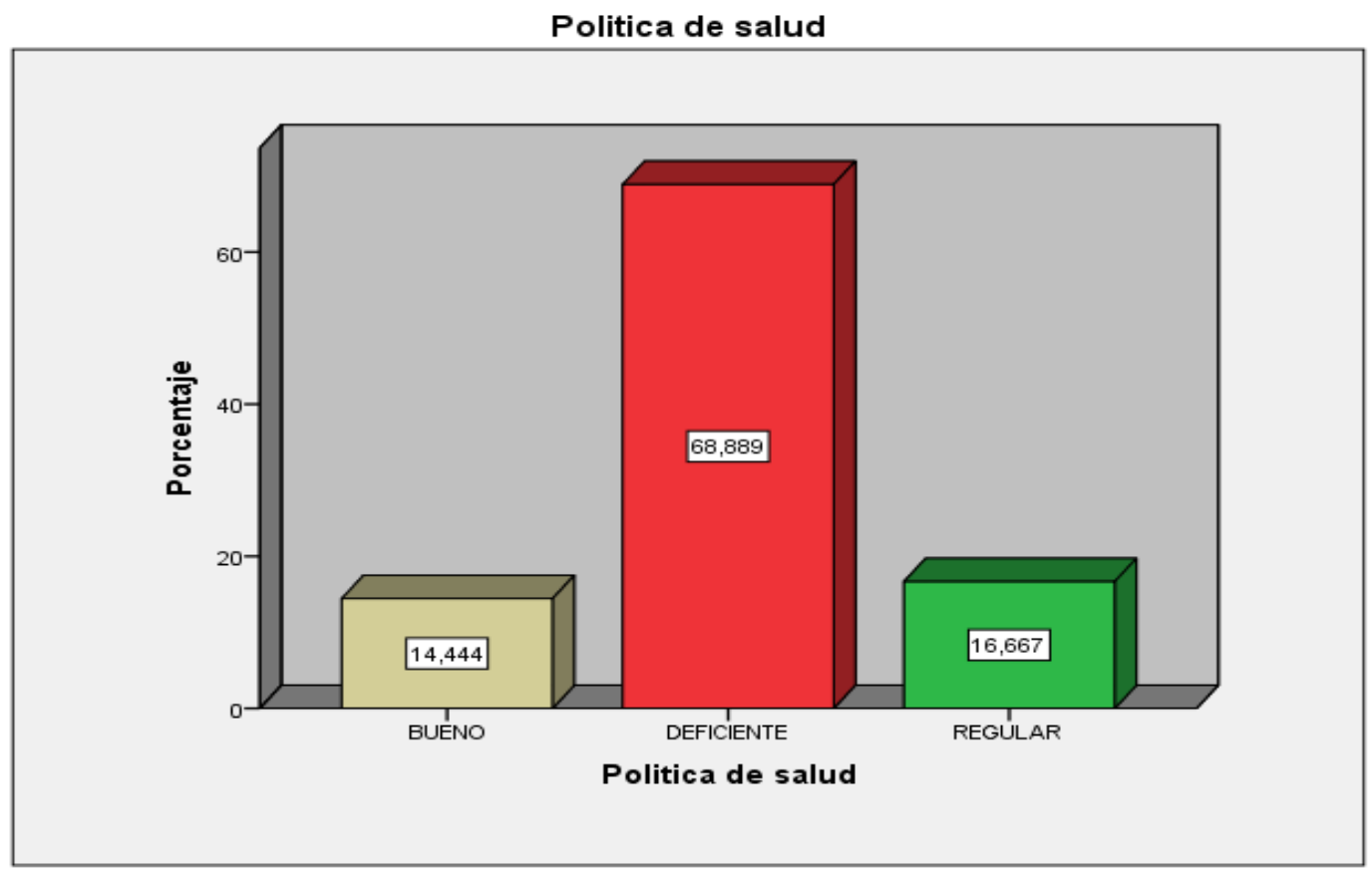

De la tabla 1 y figura 1 , se observan el $68.89 \%$ de los trabajadores en el cono norte consideran que la política de salud es deficiente, en cambio el $14,44 \%$ de los mismos consideraron que la política de salud es buena. 


\section{Tabla 2}

Dimensión política de salud equitativa frente a la Covid-19 del cono norte

\begin{tabular}{clcc}
\hline & & Frecuencia & Porcentaje \\
\hline \multirow{3}{*}{ Válido } & Bueno & 13 & 14,4 \\
& Deficiente & 54 & 60,0 \\
& Regular & 23 & 25,6 \\
& Total & 90 & 100,0 \\
\hline
\end{tabular}

\section{Figura 2}

Dimensión política de salud equitativa frente a la Covid-19 del cono norte.

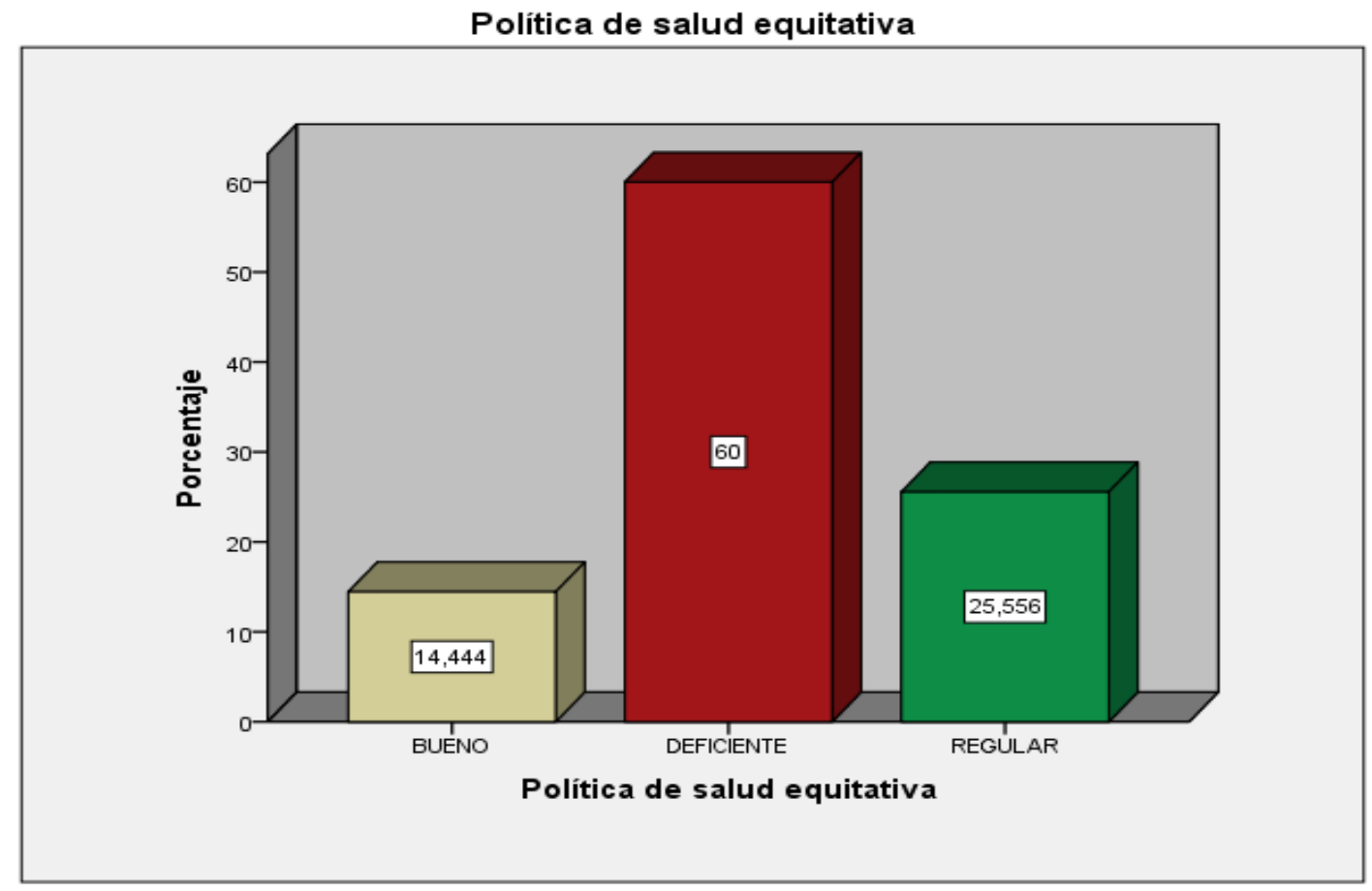

Se observan, el $60.00 \%$ de los trabajadores del cono norte consideran que la política de salud equitativa es deficiente, mientras que el 14,44\% de los mismos consideran que la política de salud equitativa es buena. 


\section{Tabla 3}

Niveles de la dimensión política de salud en caso de pandemia frente a la Covid-19 del cono norte - Lima.

\begin{tabular}{|c|c|c|c|}
\hline & & & Porcentaje \\
\hline \multirow{4}{*}{ Válido } & Bueno & 13 & 14,4 \\
\hline & Deficiente & 49 & 54,4 \\
\hline & Regular & 28 & 31,1 \\
\hline & Total & 90 & 100,0 \\
\hline
\end{tabular}

\section{Figura 3}

Dimensión política de salud en caso de pandemia frente a la Covid-19 del cono norteLima.

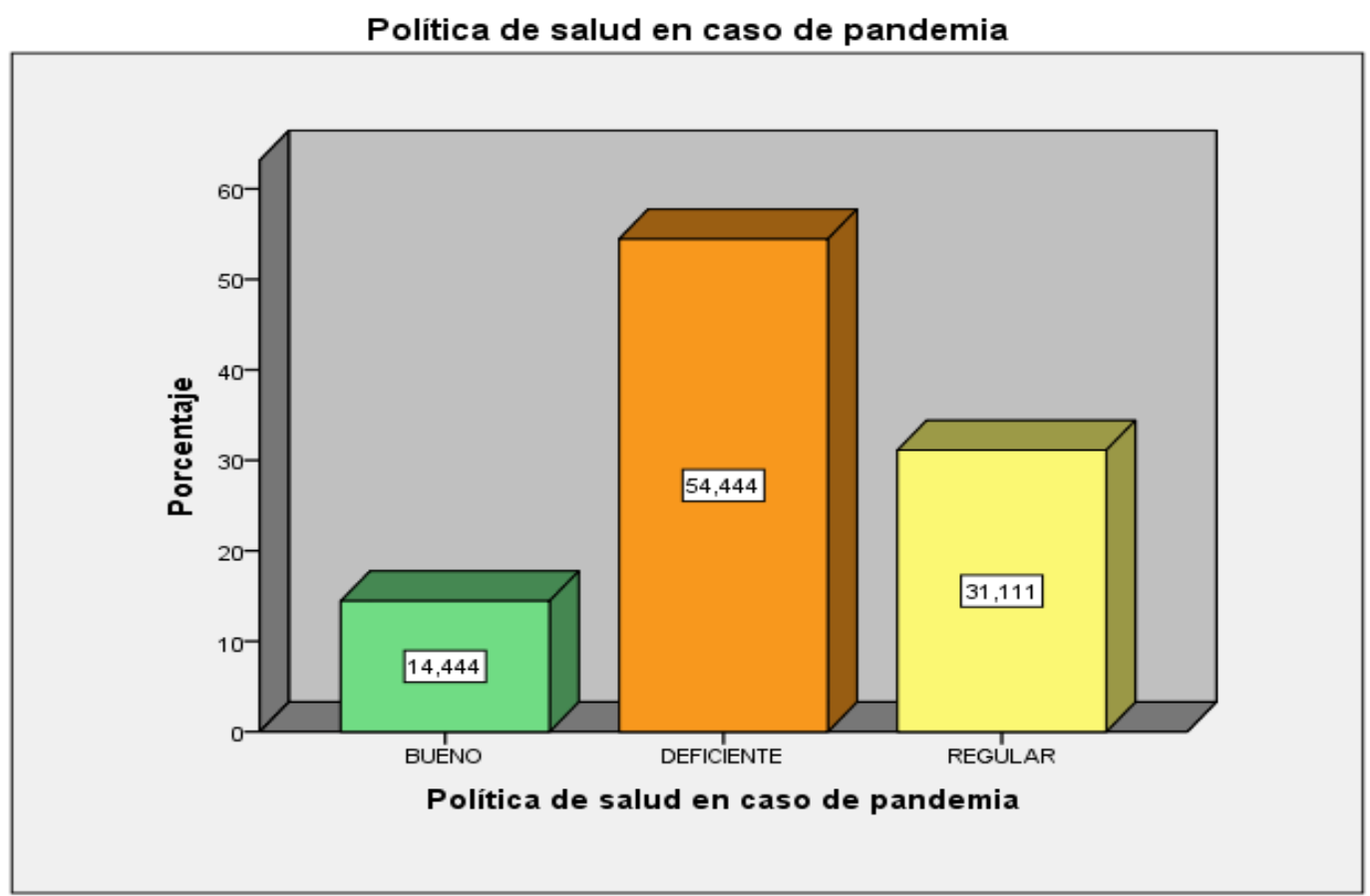

Se observan el 54.44\% de los trabajadores del cono norte consideran que la política de salud en caso de pandemia es deficiente, en cambio el 14,44\% de los mismos consideran que son buenas. 
Politica de salud y crisis sanitaria...

\section{Tabla 4}

Dimensión gasto público en salud en caso de pandemia frente a la Covid-19 del cono norte - Lima

\begin{tabular}{llcc}
\hline & & Frecuencia & Porcentaje \\
\hline \multirow{3}{*}{ Válido } & Bueno & 13 & 14,4 \\
& Deficiente & 64 & 71,1 \\
& Regular & 13 & 14,4 \\
& Total & $\mathbf{9 0}$ & $\mathbf{1 0 0 , 0}$ \\
\hline
\end{tabular}

\section{Figura 4}

Niveles de la dimensión gasto público en salud en caso de pandemia frente a la Covid19 del cono norte - Lima.

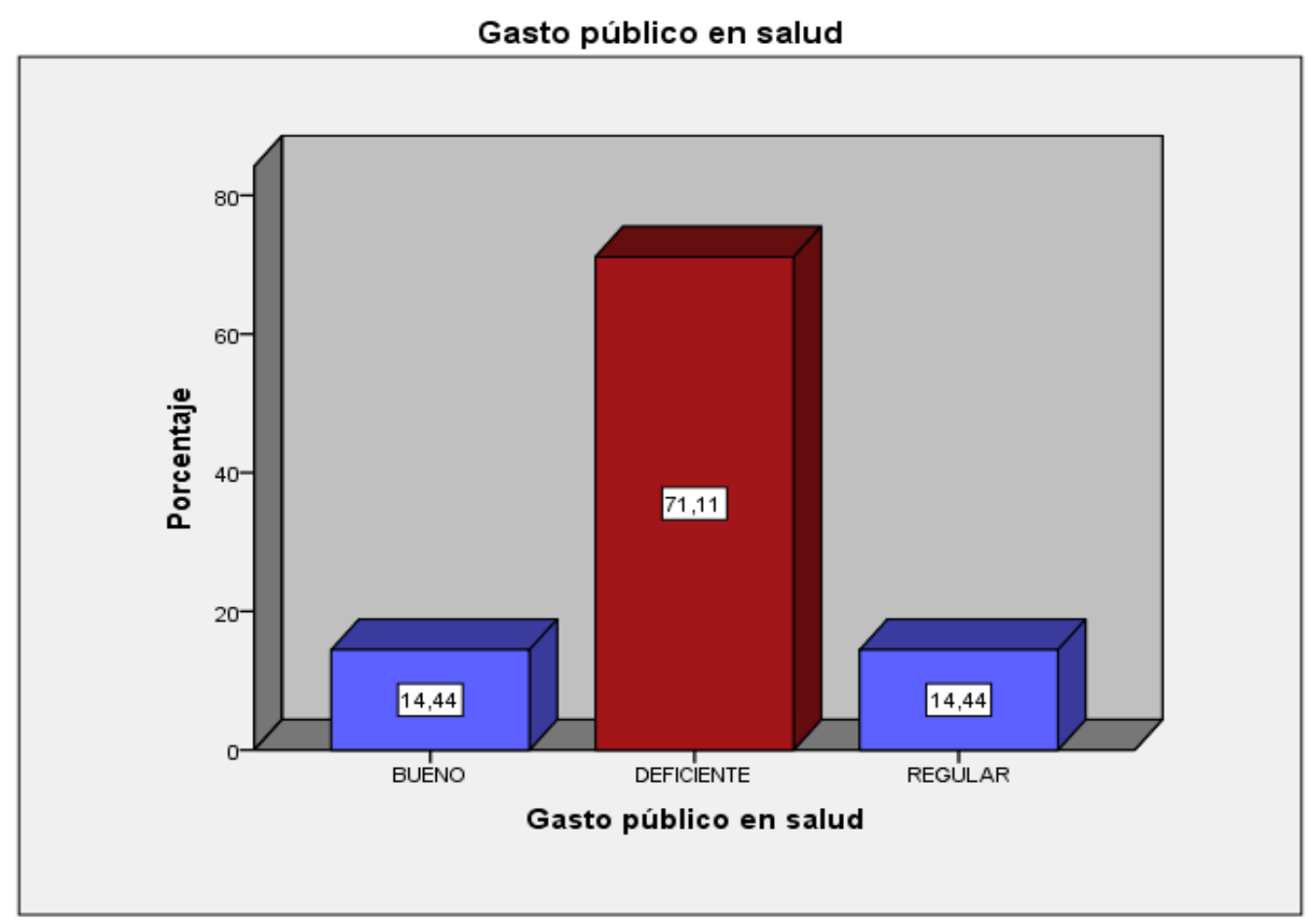

Se observa el 71,11\% de los trabajadores del cono norte consideran que gasto público en salud es deficiente, mientras que el $14,44 \%$ de los mismos consideran que la es bueno. 


\section{Tabla 5}

Niveles de la variable crisis sanitaria frente a la Covid-19 del cono norte - Lima.

\begin{tabular}{llcc}
\hline & & Frecuencia & Porcentaje \\
\hline \multirow{3}{*}{ Válido } & Bueno & 13 & 14,4 \\
& Deficiente & 58 & 64,4 \\
& Regular & 19 & 21,1 \\
& Total & $\mathbf{9 0}$ & $\mathbf{1 0 0 , 0}$ \\
\hline
\end{tabular}

\section{Figura 5}

Niveles de la variable crisis sanitaria frente a la Covid-19 del cono norte - Lima.

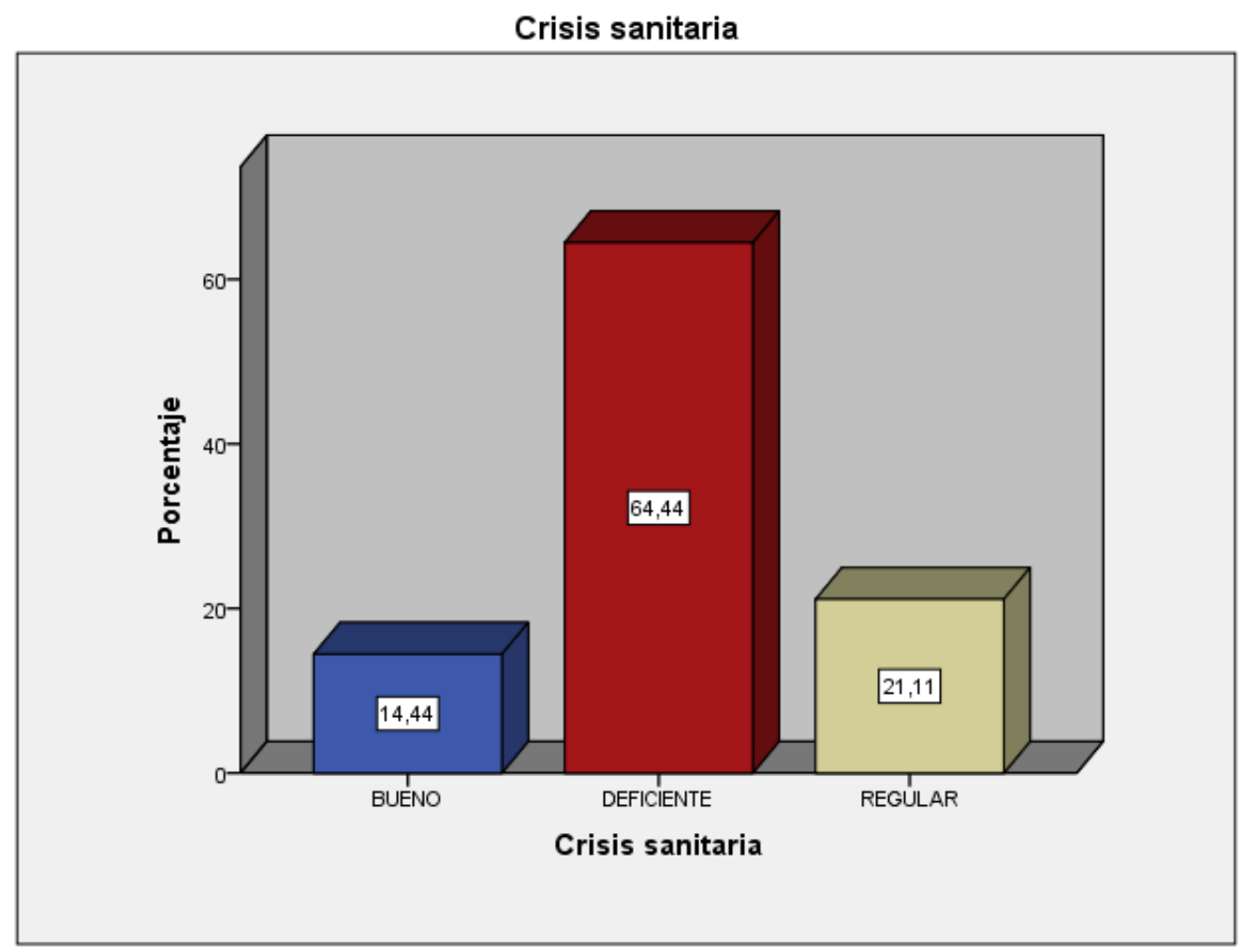

Se observa el $64,44 \%$ de los trabajadores del cono norte consideran que crisis sanitaria es deficiente, mientras que el $14,44 \%$ de los mismos consideran que la es bueno. 
Politica de salud y crisis sanitaria $\ldots$

\section{Tabla 6}

Cruzada de las variables política de salud y crisis sanitaria frente a la Covid-19 del cono norte - Lima

Tabla de cruzada Política de salud * Crisis sanitaria

\begin{tabular}{|c|c|c|c|c|c|c|}
\hline & \multicolumn{3}{|c|}{ Crisis sanitaria } & \multirow{2}{*}{ Total } \\
\hline & & & ALTO & ВАJO & MEDIO & \\
\hline \multirow{9}{*}{$\begin{array}{l}\text { Política } \\
\text { de salud }\end{array}$} & \multirow{3}{*}{ Bueno } & Recuento & 11 & 9 & 13 & 33 \\
\hline & & $\%$ del total & $12,2 \%$ & $10,0 \%$ & $14,4 \%$ & $36,7 \%$ \\
\hline & & Residuos tipificados & $-2,2$ & ,8 & 3,8 & \\
\hline & \multirow{3}{*}{ Deficiente } & Recuento & 12 & 0 & 0 & 12 \\
\hline & & $\%$ del total & $13,3 \%$ & $0,0 \%$ & $0,0 \%$ & $13,3 \%$ \\
\hline & & Residuos tipificados & 1,5 & $-1,6$ & $-1,3$ & \\
\hline & \multirow{3}{*}{ Regular } & Recuento & 35 & 10 & 0 & 45 \\
\hline & & $\%$ del total & $38,9 \%$ & $11,1 \%$ & $0,0 \%$ & $50,0 \%$ \\
\hline & & Residuos tipificados & 1,1 & ,2 & $-2,5$ & \\
\hline \multirow{2}{*}{ Total } & & Recuento & 58 & 19 & 13 & 90 \\
\hline & & $\%$ del total & $64,4 \%$ & $21,1 \%$ & $14,4 \%$ & $100,0 \%$ \\
\hline
\end{tabular}

\section{Figura 6}

Tabla Cruzada de las variables política de salud y crisis sanitaria frente a la Covid-19 del cono norte - Lima.

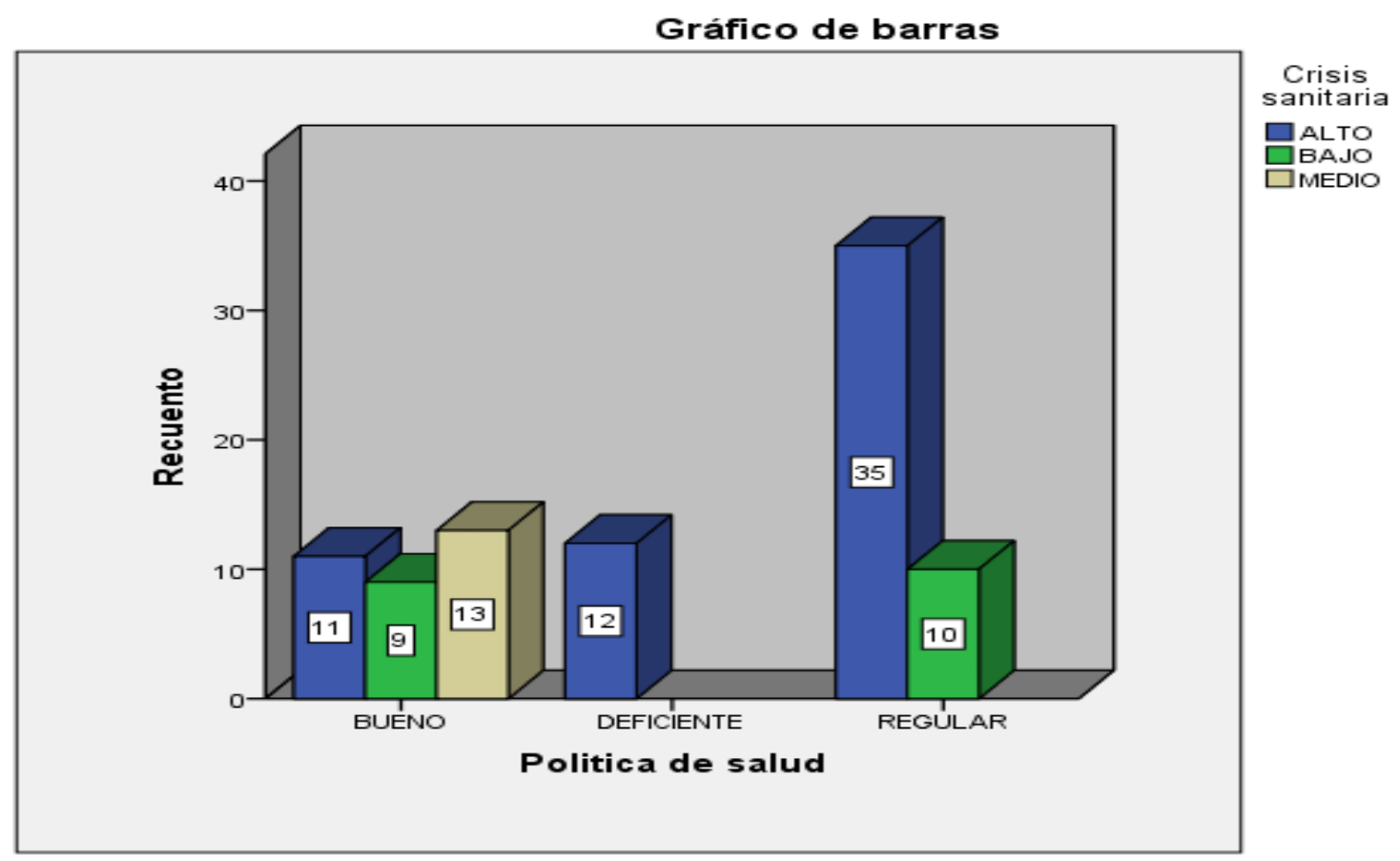


Se observa el $35.00 \%$ de los trabajadores que consideran una regular política de salud también consideran una alta crisis sanitaria, el $13.00 \%$ de los trabajadores que consideran una buena política de salud, además consideran un nivel medio de crisis sanitaria, en cambio el $10.00 \%$ de los trabajadores que consideran una regular política de salud también consideran un nivel bajo de crisis sanitaria.

\section{Prueba de hipótesis}

Para contrastar las hipótesis se probó utilizando la correlación de Spearman.

\section{Hipótesis general}

\section{Hipótesis de investigación}

Ho : La política de salud no se relaciona significativamente con la crisis sanitaria frente a la Covid-19 del cono norte - Lima.

Ha : La política de salud se relaciona significativamente con la crisis sanitaria frente a la Covid-19 del cono norte - Lima.

\section{Tabla 7}

Coeficiente de correlación entre la política de salud y crisis sanitaria frente a la Covid19 del cono norte - Lima.

\section{Correlaciones}

\begin{tabular}{|c|c|c|c|c|}
\hline & & & $\begin{array}{r}\mathrm{Cl} \\
\text { san }\end{array}$ & \\
\hline \multirow[t]{3}{*}{ Rho de Spearman } & Política de salud & Coeficiente de correlación & & $0.928^{*+}$ \\
\hline & & Sig. (bilateral) & & 0,000 \\
\hline & & $\mathrm{N}$ & 90 & 90 \\
\hline
\end{tabular}

Se observa el resultado de la aplicación del coeficiente de correlación Rho de Sperman para determinar la relación entre política de salud y crisis sanitaria, se indicó un índice de $\mathrm{r}=0.928^{* *}$, la significancia bilateral es $\mathrm{p}=0.000$ es menor a 0.05 , concluyendo la existencia de una correlación positiva muy alta entre las dos variables en consecuencia la relación es significativa y se rechaza la hipótesis nula (H0) asumiendo que la política de salud se relaciona significativamente con la crisis sanitaria frente a la Covid-19 del cono norte - Lima. 


\section{Primera hipótesis específica}

\section{Hipótesis de investigación}

Ho : La dimensión política de salud equitativa no se relaciona significativamente con la crisis sanitaria frente a la Covid-19 del cono norte - Lima.

Ha : La dimensión política de salud equitativa se relaciona significativamente con la crisis sanitaria frente a la Covid-19 del cono norte - Lima.

\section{Tabla 8}

Coeficiente de correlación entre política de salud equitativa y crisis sanitaria frente a la Covid-19 del cono norte - Lima.

\begin{tabular}{lllrr}
\hline \hline & & & $\begin{array}{c}\text { D1 Política de } \\
\text { salud } \\
\text { equitativa }\end{array}$ & $\begin{array}{c}\text { V2 Crisis } \\
\text { sanitaria }\end{array}$ \\
\hline Rho de Spearman & $\begin{array}{c}\text { D1 Política de salud } \\
\text { equitativa }\end{array}$ & Coeficiente de correlación & 1.000 & $.645^{* *}$ \\
& Sig. (bilateral) & N & .000 & 90 \\
\cline { 2 - 5 } & V2 Crisis sanitaria & Coeficiente de correlación &, $645^{-*}$ & 1,000 \\
& Sig. (bilateral) &, 000 & 90 \\
\hline \hline
\end{tabular}

**. La correlación es significativa en el nivel 0,01 (bilateral).

Se observa el resultado de la aplicación del coeficiente de correlación Rho de Sperman para determinar la relación política de salud equitativa y crisis sanitaria, se indicó un índice de $\mathrm{r}=0.645^{* *}$, la significancia bilateral es $\mathrm{p}=0.000$ es menor a 0.05 , concluyendo la existencia de una correlación positiva moderada en consecuencia la relación es significativa y se rechaza la hipótesis nula(H0) asumiendo que la dimensión política de salud equitativa se relaciona significativamente con la crisis sanitaria frente a la Covid19 del cono norte - Lima.

\section{Segunda hipótesis específica}

\section{Hipótesis de investigación}

Ho : La dimensión política de salud en caso de pandemia no se relaciona significativamente con la crisis sanitaria frente a la Covid-19 del cono norte - Lima. 
Ha : La dimensión política de salud en caso de pandemia se relaciona significativamente con la crisis sanitaria frente a la Covid-19 del cono norte - Lima.

\section{Tabla 9}

Coeficiente de correlación entre política de salud en caso de pandemia y crisis sanitaria frente a la Covid-19 del cono norte - Lima.

\begin{tabular}{lllrr}
\hline \hline & & & $\begin{array}{c}\text { D1 Política de } \\
\text { salud en caso } \\
\text { de pandemia }\end{array}$ & $\begin{array}{c}\text { V2 Crisis } \\
\text { sanitaria }\end{array}$ \\
\hline Rho de Spearman & $\begin{array}{c}\text { D1 Política de salud } \\
\text { en caso de pandemia }\end{array}$ & $\begin{array}{c}\text { Coeficiente de correlación } \\
\text { Sig. (bilateral) }\end{array}$ & 1.000 & $.758^{* *}$ \\
& $\mathrm{~N}$ & 900 &, 000 \\
\cline { 2 - 5 } & V2 Crisis sanitaria & Coeficiente de correlación &, $758^{-*}$ & 1,000 \\
& Sig. (bilateral) &, 000 & 90 \\
& $\mathrm{~N}$ & 90 & 90 \\
\hline \hline
\end{tabular}

**. La correlación es significativa en el nivel 0,01 (bilateral).

Se observa el resultado de la aplicación del coeficiente de correlación Rho de Sperman para determinar la relación entre política de salud en caso de pandemia y crisis sanitaria, se indicó un índice de $0.758^{* *}$, la significancia bilateral es $\mathrm{p}=0.000$ es menor a 0.05 , concluyendo la existencia de una correlación positiva alta, en consecuencia la relación es significativa y se rechaza la hipótesis nula (H0) asumiendo que la dimensión política de salud en caso de pandemia se relaciona significativamente con la crisis sanitaria frente a la Covid-19 del cono norte - Lima. .

\section{Tercera hipótesis específica}

\section{Hipótesis de investigación}

H0 : La dimensión gasto público en salud no se relaciona significativamente con la crisis sanitaria frente a la Covid-19 del cono norte - Lima.

Ha : La dimensión gasto público en salud se relaciona significativamente con la crisis sanitaria frente a la Covid-19 del cono norte - Lima. 


\section{Tabla 10}

Coeficiente de correlación entre gasto público en salud y crisis sanitaria frente a la Covid-19 del cono norte - Lima.

\begin{tabular}{|c|c|c|c|c|}
\hline & & & $\begin{array}{c}\text { D1 Gasto } \\
\text { público en } \\
\text { salud }\end{array}$ & $\begin{array}{l}\text { V2 Crisis } \\
\text { sanitaria }\end{array}$ \\
\hline \multirow[t]{5}{*}{ Rho de Spearman } & $\begin{array}{l}\text { D1 Gasto público en } \\
\text { salud }\end{array}$ & $\begin{array}{l}\text { Coeficiente de correlación } \\
\text { Sig. (bilateral) }\end{array}$ & 1.000 & $\begin{array}{r}.901^{-*} \\
.000\end{array}$ \\
\hline & & $\mathrm{N}$ & 90 & 90 \\
\hline & V2 Crisis sanitaria & Coeficiente de correlación &, $901^{-}$ & 1,000 \\
\hline & & Sig. (bilateral) &, 000 & . \\
\hline & & $\mathrm{N}$ & 90 & 90 \\
\hline
\end{tabular}

**. La correlación es significativa en el nivel 0,01 (bilateral).

Se observa el resultado de la aplicación del coeficiente de correlación Rho de Sperman para determinar la relación entre gasto público en salud y crisis sanitaria, se indicó un índice de $0.901^{* *}$, la significancia bilateral es $\mathrm{p}=0.000$ es menor a 0.05 , concluyendo la existencia de una correlación positiva muy alta, en consecuencia la relación es significativa y se rechaza la hipótesis nula (H0) asumiendo que la dimensión gasto público en salud se relaciona significativamente con la crisis sanitaria frente a la Covid-19 del cono norte - Lima.

\section{DISCUSIÓN}

El dictamen del estudio doctoral referente a política de salud y crisis sanitaria frente a la Covid-19 del cono norte, mediante la aplicación de encuesta a los trabajadores, ha permitido obtener un análisis estadístico destallado que se muestra en las gráficas y tablas con resultados de $0,928 \%$, siendo positiva muy alta. Por consecuente, la crisis sanitaria exige al gobierno a tomar medidas que cambien el patrón del escenario actual. Teniendo en cuanta, Zhang et al. (2021), en su artículo titulado: "Disparidades políticas en respuesta a la primera oleada de COVID-19 entre China y Alemania”. Objetivo: Nuestra investigación resumió las disparidades políticas en respuesta a la primera ola de COVID19 entre China y Alemania. Esperamos aportar experiencia política a otros países que aún se encuentran en epidemias graves. Métodos: Utilizamos un modelo lineal generalizado para evaluar las asociaciones entre las principales políticas de control y el número de 
casos confirmados y las disparidades de políticas en respuesta a la primera ola de COVID19 entre China y Alemania. Resultados: Los modelos lineales generalizados muestran que los siguientes factores influyen para números acumulado confirmado para China: Mecanismo Conjunto de Prevención y Control; el bloqueo de las zonas más afectadas; la respuesta de más alto nivel a las emergencias de salud pública; la ampliación de la cobertura del seguro médico a los pacientes sospechosos; los hospitales improvisados; la gestión residencial cerrada; la asistencia de contraparte. Concluyendo, hay dos diferencias entre China y Alemania en las intervenciones no farmacéuticas: China adoptó la estrategia de bloqueo, y Alemania adoptó la estrategia de mitigación primero y de bloqueo después; el objetivo de China es eliminar el virus, y el de Alemania es proteger a los grupos de alto riesgo para reducir las pérdidas. Al mismo tiempo, las políticas aplicadas por ambos países tienen similitudes: el bloqueo estricto es una medida clave para controlar la fuente de infección, y la mejora de la capacidad de respuesta médica es una forma importante de reducir la mortalidad.

Por lo tanto, de acuerdo Wang et al. (2021), en su artículo titulado: "Disparidades políticas en la lucha contra el COVID-19 entre Japón, Italia, Singapur y China": Objetivo: Con el fin de aportar experiencias para el control de epidemias a nivel internacional, este estudio resumió sistemáticamente las políticas de prevención y controles de enfermedades por COVID-19 en Japón, Italia, China y Singapur, Resumimos políticas en prevención y controles de epidemia tanto Japón, Italia, China y Singapur, y analizamos los efectos de las políticas de estos cuatro países utilizando los datos publicados. Resultados: A partir del 27 de mayo de 2020, la tendencia creciente de nuevos casos en Japón, Italia, China y Singapur se ha estabilizado. Sin embargo, el número acumulado de casos confirmados (231139) y la tasa de letalidad (14,3\%) en Italia superaron con creces los de los otros tres países, y el efecto del control de la epidemia fue inferior. Según los resultados del control de la epidemia de estos cuatro países, podemos concluir que las medidas de bloqueo fueron en general eficaces. Como estrategia central de las medidas de bloqueo, la admisión de pacientes leves en el hospital y el rastreo de casos ayudaron a frenar la propagación del brote en Singapur y China. Los países deben elegir las estrategias de respuesta adecuadas bajo la premisa de considerar su propia situación, aumentar la inversión en recursos sanitarios para garantizar la equidad sanitaria mundial y, finalmente, controlar la propagación de las enfermedades infecciosas en el mundo de 
forma eficaz. Citando a Wang et al. (2021), en su artículo titulado: “Gobernanza de la resiliencia comunitaria en la crisis de salud pública en China": Objetivo: Explorar las manifestaciones y el mecanismo de formación del problema de la resiliencia insuficiente de gobernanza en crisis de salud públicas de la comunidad, basándonos en la teoría del sistema adaptativo complejo, que hace hincapié en la interacción entre los sujetos y entre los sujetos y el entorno para mejorar la adaptabilidad al entorno. Se realizaron cuestionarios y entrevistas en profundidad en 28 condados (distritos) de 14 ciudades de 7 provincias de China; se recogieron 2.345 cuestionarios y 71 datos de entrevistas, y realizamos un análisis estadístico descriptivo de los datos de los cuestionarios. Se constató que algunas comunidades se enfrentaban a problemas de resiliencia insuficientes, como el "simple aislamiento de los hogares y las comunidades", el "establecimiento ciego de límites", la "ley por capas" y el "rechazo y repulsión de todos los individuos procedentes de Hubei o incluso relacionados con ella". Conclusión: Se encontró que en la lucha contra el COVID-19 en China, el gobierno chino ha controlado eficazmente la propagación de la pandemia de COVID-19, y las comunidades de base frente a su papel importantes de lucha frente al COVID-19, pero algunas comunidades carecen de fuerza para llevar a cabo una labor de prevención y control independiente y flexible, y en su lugar se dedicaron a "simplemente cerrar los hogares y las comunidades", "establecer ciegamente los límites", "capa por capa de la ley", y "rechazar y repeler a todos los individuos de Hubei o incluso relacionados con ella", lo que es una manifestación típica de la insuficiente capacidad de recuperación de la comunidad. En la actual situación de alto riesgo, para lograr una verdadera gobernanza resiliente de crisis de salud pública de la comunidad, es necesario centrarse en la comunidad.

Desde la posición de Nosyk et al. (2021), en su estudio de investigación: "Evaluación de las medidas de mitigación del riesgo para la persona con trastorno por consumos en sustancia haciendo frentes en doble crisis de salud pública en pandemia, la sobredosis en Columbia Británica: Un protocolo de estudio de método mixto". Al comienzo de estas dos emergencias de salud pública, el gobierno de la Columbia Británica anunció una Guía de Mitigación de Riesgos (RMG) provisional que permitía prescribir alternativas de medicación a las sustancias, incluidos los opioides, el alcohol, los estimulantes y las benzodiacepinas, una intervención a veces denominada "suministro seguro". Métodos y análisis Realizamos un estudio paralelo de métodos mixtos que incluía tanto el análisis 
de datos sanitarios administrativos a nivel de población como la recopilación de datos primarios, incluyendo un estudio observacional longitudinal de 10 semanas (objetivo $\mathrm{n}=200$ ), una encuesta transversal (objetivo $\mathrm{n}=200$ ) y entrevistas cualitativas (objetivo $\mathrm{n}=60$ ). Hemos aplicado un enfoque participativo a esta evaluación, colaborando con personas que han vivido o viven el consumo de drogas, e investigadores y responsables de la salud pública de toda la provincia. BC con indicación de SUD entre 1996 y 2020. A juicio de Link (2021), en su estudio de investigación: "Evitación de la información durante las crisis sanitarias: Predictores de la evitación de información: Objetivo: Este estudio investiga la prevalencia de la evitación de la información específica de la fuente entre los consumidores alemanes y los predictores del comportamiento de evitación de la información. Utilizando una muestra demográfica estratificada de consumidores de noticias de un estado federal alemán $(\mathrm{N}=1.000)$, examinamos empíricamente el modelo propuesto, respondemos a la pregunta de investigación y ponemos a prueba nuestras hipótesis utilizando un modelo de ecuaciones estructurales. Conclusión: Este estudio proporciona información sobre el desarrollo de la teoría, contribuye a la literatura sobre el comportamiento de la información e identifica las barreras de las comunicaciones en crisis sanitaria. Por lo tanto, Ding et al. (2020), en su estudio: "Percepción del riesgo y depresión en la crisis de salud pública: Prueba en crisis frente al covid-19 de China": Los objetivos del estudio son (1) construir un marco conceptual para la percepción del riesgo y la depresión de las personas en las crisis de salud públicas, (2) examinar cómo la salud mental de las personas mediante crisis de la enfermedades del coronavirus 2019, se ve afectada por la percepción del riesgo y sus factores asociados, incluyendo la percepción a distancia de la crisis (3) proponer recomendaciones políticas sobre cómo tratar los problemas psicológicos en la actual crisis de COVID-19. Métodos: Se aplicó una encuesta con cuestionario en línea. Un total de 6373 personas visitaron el cuestionario en línea, 1115 personas completaron el cuestionario, y el número de cuestionarios válidos fue de 1081. Se empleó los modelos de ecuación estructural en análisis para datos. Concluyendo el importante papel en hora para afectar salud mental en personas frente a crisis de salud pública. Por lo tanto, las políticas sanitarias destinadas a mejorar el bienestar psicológico de las personas en una crisis de salud pública deberían tener en cuenta la percepción del riesgo. 2020 por los autores. Licencia MDPI, Basilea, Suiza. Mendoza et al. (2018) en su artículo titulado: "El proceso de reforma del sector salud en Perú". Objetivo. 
Caracterización de los procesos mediante reformas del sector salud, del Perú (lanzado oficialmente el 2013), teniendo como principal reto proporcionar coberturas de aseguramientos no relacionadas mediante pobreza en la población, disponiendo de abundantes recursos humanos para satisfacer al ciudadano frente a sus demandas.

Para finalizar, en políticas sanitarias, se deben evaluar los siguientes indicadores: Recursos humanos, Organización eficiente, Eficiencia sanitaria, Gestión logística, Innovación, cumplimiento, Presupuestos que tienen injerencia directa y permitiendo examinar la situación real de las políticas sanitarias establecidas por el gobierno en favor del ciudadano frente a la Covid-19 del cono norte - Lima.

\section{CONCLUSIÓN O CONSIDERACIONES FINALES}

Se logra determina que existencia de correlación positiva muy alta entre política de salud y crisis sanitaria frente a la Covid-19 del cono norte - Lima. Los resultados obtenidos indican una correlación de Sperman $\mathrm{R}=0,928^{* *}$, con una significancia de 0.000 que es menor al $1 \%$ de significancia estándar $(\mathrm{P}<0.01)$, determinando correlación positiva muy alta.

Logra determina que existe una correlación confiable entre política de salud equitativa y crisis sanitaria frente a la Covid-19 del cono norte - Lima. Los resultados obtenidos indican una correlación de Sperman $\mathrm{R}=0,645^{* *}$, con una significancia de 0.000 que es menor al $1 \%$ de significancia estándar $(\mathrm{P}<0.01)$, determinando correlación confiable en ambas.

Se logra determina que existe una correlación confiable entre política de salud en caso de pandemia y crisis sanitaria frente a la Covid-19 del cono norte - Lima. Los resultados obtenidos indican una correlación de Sperman $\mathrm{R}=0,758^{* *}$, con una significancia de 0.000 que es menor al $1 \%$ de significancia estándar $(\mathrm{P}<0.01)$, determinando correlación confiable en ambas.

Se logra determina que existe una correlación confiable entre gasto público en salud y crisis sanitaria frente a la Covid-19 del cono norte - Lima. Los resultados obtenidos indican una correlación de Sperman $\mathrm{R}=0,901^{* *}$, con una significancia de 0.000 que es menor al $1 \%$ de significancia estándar $(\mathrm{P}<0.01)$, determinando excelente correlación de confiablidad en ambas. 


\section{LISTA DE REFERENCIAS}

Ding, Y., Xu, J., Huang, S., Li, P., Lu, C., \& Xie, S. (2020). Risk perception and depression in public health crises: Evidence from the covid-19 crisis in china. International Journal of Environmental Research and Public Health, 17(16), 1-17. doi:10.3390/ijerph17165728

Garcia-Casanovas, A., Bisbe, E., Colomina, M. J., Arbona, C., \& Varela, J. (2020). Health policy strategies for patient blood management implementation throughout the Spanish health systems. [Estrategias de gestión y política sanitaria para el desarrollo del Patient Blood Management en los sistemas de salud en España] Journal of Healthcare Quality Research, 35(5), 319-327. doi: 10.1016/j.jhqr.2020.06.005

Gough, I. (2003). Capital global, necesidades básicas y política social. Madrid: Nino y Davila.

Hernández, R., Fernández, C., y Baptista, L. (2010). Metodología de la investigación.

Ibáñez Peiró, A. (2020). La actividad informativa del Gobierno español durante la emergencia sanitaria provocada por el coronavirus, COVID-19. Revista Española de Comunicación en Salud, sup. 1, S304-318. doi: 10.20318/recs.2020.5441

Ibáñez Peiró, A. (2020). La actividad informativa del Gobierno español durante la emergencia sanitaria provocada por el coronavirus, COVID-19. Revista Española de Comunicación en Salud, sup. 1, S304-318. doi: 10.20318/recs.2020.5441

Janssen, M., \& van der Voort, H. (2020). Agile and adaptive governance in crisis response_Lessons from the COVID-19 pandemic| Elsevier Enhanced Reader. International Journal of Information Management. https://doi.org/https://doi.org/10.1016/j.ijinfomgt.2020.102180

Lahera, Eugenio (2006). Del dicho al hecho: ¿cómo implementar las políticas? En Revista del CLAD Reforma y Democracia, No. 35, junio, Caracas.

Lamata Cotanda, F. (2006). Crisis sanitarias y respuesta política. administración sanitaria, $387-569$.

Link, E. (2021). Information avoidance during health crises: Predictors of avoiding information about the COVID-19 pandemic among german news consumers. Information Processing and Management, 58(6) doi: 10.1016/j.ipm.2021.102714 
Llerena, R., \& Sánchez, C. (20 de abril de 2020). Emergencia, gestión, vulnerabilidad y respuestas frente al impacto de la pandemia COVID19 en el Perú.

Mendoza-Arana, P. J., Rivera-Del Río, G., Gutiérrez-Villafuerte, C., \& SanabriaMontáñez, C. (2018). The process of health sector reform in Perú. [El proceso de reforma del sector salud en Perú] Revista Panamericana De Salud Pública/Pan American Journal of Public Health, 42 doi:10.26633/RPSP.2018.74: https://repositorio.ucv.edu.pe/

Minsa (2011). Documento técnico: Lineamientos de política de promoción de salud en el Perú RM No 464-2011. Lima, Perú: chataro E.R.L.

Nosyk, B., Slaunwhite, A., Urbanoski, K., Hongdilokkul, N., Palis, H., Lock, K., . . . Pauly, B. (2021). Evaluation of risk mitigation measures for people with substance use disorders to address the dual public health crises of COVID-19 and overdose in british columbia: A mixed-method study protocol. BMJ Open, 11(6) doi:10.1136/bmjopen-2020-048353

Oliveira, A. P. C., Poz, M. R. D., Craveiro, I., Gabriel, M., \& Dussault, G. (2018). Factors that influence human resources for health policy formulation: A multiple case study in brazil and portugal. [Fatores que influenciaram o processo de formulação de políticas de recursos humanos em saúde no Brasil y em Portugal: estudo de caso múltiplo] Cadernos De Saude Publica, 34(2), e00220416. doi:10.1590/0102$311 \mathrm{X} 00220416$

Parodi Trece, C. (2005). Economía de las Políticas Sociales. Lima: Universidad del Pacífico. https://core.ac.uk/download/pdf/51208859.pdf

Sierra Rodríguez, J. (2020). Periodismo, comunicación institucional y transparencia: aprendizajes de la crisis sanitaria del COVID-19. Revista de Comunicación y Salud, 10 (2), 569-591. doi: https://doi.org/10.35669/rcys.2020.10(2).569-591

Testa M. Pensar en Salud. Buenos Aires: Lugar Editorial,1993:99.

Tobar, F (2012)." Políticas de salud: Conceptos y herramientas" En: GARAY, O (Coordinador) quiere actualizar el libro (collectives; que yo coordine) llamado "Responsabilidad Profesional de los Médicos. Ética, Bioética y Jurídica. Civil y Penal". Buenos Aires. La Editorial La Ley. 2012 
Wang, C., Dong, X., Zhang, Y., \& Luo, Y. (2021). Community resilience governance on public health crisis in china. International Journal of Environmental Research and Public Health, 18(4), 1-20. doi:10.3390/ijerph18042123

Wang, X., Shi, L., Zhang, Y., Chen, H., \& Sun, G. (2021). Policy disparities in fighting Covid-19 among japan, italy, singapore and china. International Journal for Equity in Health, 20(1) doi:10.1186/s12939-020-01374-2

Westlund, O. y Ghersetti, M. (2015). Modelling news media use. Positing and applying the $\mathrm{GC} / \mathrm{MC}$ model to the analysis of media use in everyday life and crisis situations. Journalism studies, 16(2), 133-151. doi: 10.1080/1461670X.2013.868139

Zhang, Y., Shi, L., Chen, H., Wang, X., \& Sun, G. (2021). Policy disparities in response to the first wave of COVID-19 between china and germany. International Journal for Equity in Health, 20(1) doi:10.1186/s12939-021-01424-3 